\title{
PROPUESTA DE EVALUACIÓN DE RIESGOS EMPRESARIALES EN MICROEMPRESAS MANUFACTURERAS
}

\author{
PROPOSED RISK ASSESSMENT IN MICRO MANUFACTURING BUSINESS \\ Suly Sendy Pérez Castañeda* \\ Dorie Cruz Ramírez*
}

Universidad Autónoma del Estado de Hidalgo-UAEH / Hidalgo - México

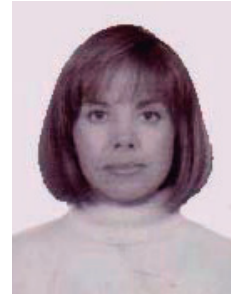

[Recepción: Marzo de 2014/ Conformidad: Mayo 2014]

\section{RESUMEN}

$\mathrm{Al}$ reconocer que las micro empresas representan una parte esencial del tejido empresarial nacional e internacional por su contribución al empleo y a la generación de riqueza, estas se encuentran sometidas a riesgos que, si no son adecuadamente gestionados, afectan no solo su crecimiento, sino incluso amenazan su permanencia, por lo que se hace indispensable identificar y medir el nivel de exposición de riesgos en estas entidades; sin embargo, las metodologías existentes se basan en datos estadísticos que este tipo de empresas no generan.

Bajo este contexto, el estudio presenta una propuesta de evaluación del riesgo empresarial en microempresas desde una perspectiva cualitativa, basada en la metodología del Enterprise Risk Management (ERM), con el instrumento IEREM (Pérez, Campos y García, 2013), a través de un estudio de caso en el sector manufacturero metalmecánico mexicano. Los resultados muestran que el ERM es una metodología cualitativa de fácil aplicación para estas microempresas, que permite identificar gráfica y jerárquicamente los riesgos de mayor nivel de exposición.

Palabras clave:

Riesgo empresarial; ERM; evaluación cualitativa.

\begin{abstract}
Recognizing that micro companies are an essential part of national and international business community for his contribution to employment and wealth generation, these are subject to risks that, if not properly managed, affect not only growth, but also threaten his residence, so it is essential to identify and measuring the level of risk exposure in these institutions; however, the existing methods are based on statistical data that these businesses do not generate.

In this context, the paper presents a proposal of risk assessment in micro business from a qualitative perspective, based on the methodology of Enterprise Risk Management (ERM) in IEREM (Perez and Garcia Campos, 2013) instrument through a case study in Mexico metalworking manufacturing. The results show that ERM is a qualitative methodology easily applicable to these enterprises, which identifies chart and hierarchically in higher level of risk exposure.
\end{abstract}

\section{Keywords:}

Business risk; ERM; qualitative evaluation.

\footnotetext{
* Licenciada en Contaduría y Finanzas, Maestra en Administración de Instituciones. Email: ssendy_2005@yahoo.com.mx

** Licenciada en Contaduría, Maestra en Gestión Administrativa. Email: cruzd_r@hotmail.com

*** Colaborador: José Antonio Téllez Monroy. Estudiante de $9^{\circ}$ semestre de la Licenciatura en Contaduría, de la Universidad Autónoma del Estado de Hidalgo, México, antonioo84@hotmail.com
} 


\section{INTRODUCCIÓN}

Todos los negocios en un mercado libre se exponen al riesgo y así nace la inquietud de aplicar conceptos y técnicas sobre el riesgo que realmente tengan valor para inexpertos en la materia. Sin embargo, se observa que la gestión de los riesgos en empresas no financieras está lastrado por la ausencia de técnicas que permitan gestionar los riesgos propios de su actividad, lo que está demandando cambios respecto a lo que hasta hace poco sucedía, pero en el contexto actual, se ha demostrado que una gestión de los riesgos puede convertirse en uno de los elementos que la diferencien de la competencia y por lo tanto, la hagan permanecer y crecer en el mercado.

Por lo que este artículo está organizado en tres apartados: el primero es el marco teórico, donde se conceptualiza al ERM y los riesgos empresariales; en el segundo, se muestra la metodología aplicada para la evaluación cualitativa de los riesgos empresariales en el estudio del caso y el tercero, en donde se presentan los resultados de la evaluación.

\section{MARCO TEÓRICO}

\section{Breve contexto del ERM}

Referirse al Enterprise Risk Management (ERM) es indispensable hacer mención del Committee of Sponsoring Organizations of the Treadway Commission (COSO), organización creada en 1985 con la finalidad de mejorar la calidad de los reportes financieros, de los controles internos y el gobierno corporativo (Arnaiz, 2013). COSO proporciona un marco integral del control interno y herramientas de evaluación para sistemas de control, además de proporcionar una terminología utilizada comúnmente y principios usados como guía para desarrollar una arquitectura efectiva para la administración de riesgos, que permite una visión integral del sistema de control institucional. De aquí surge el ERM, definido como "la gestión de riesgos empresariales que consiste en un proceso de identificación y análisis de riesgo desde una perspectiva amplia e integral de la compañía" (Caicedo, 2010, p.23).

Dentro de las ventajas documentadas del uso del ERM se identifican: la ampliación de las decisiones de respuesta al riesgo; reducción de sorpresas y pérdidas operacionales; identificación y administración de riesgos a lo largo de toda la organización; proporcionar respuestas integradas a los múltiples riesgos y ayuda a la organización, para lograr objetivos y evitar pérdidas (Arnaiz, 2013; ISACA, 2009, Soldano, 2009).

\section{Conceptualización de riesgo y riesgo empresarial}

ISACA (2009) define al riesgo como "la variación que se puede producir en los resultados esperados de una situación dada, dentro de un periodo determinado" (p.2). De tal manera, que el concepto de riesgo está relacionado con la posibilidad de que ocurra un evento que se traduzca en resultados negativos o positivos para los participantes en un determinado entorno.

Bajo esta premisa, entonces el riesgo empresarial tiene su fundamento en el carácter probabilístico de la actividad empresarial, así como en la relativa incertidumbre situacional en que se desarrolla esta actividad, que tiene carácter universal (Koprinarov, 2005); es decir, que podría manifestarse y afectar a todas las etapas y sectores de la organización.

Por lo que para el presente estudio y considerando el instrumento utilizado: IEREM (Pérez, Campos y García, 2013), se evalúan los siguientes riesgos empresariales: del entorno, estratégicos, operativos y financieros. Los riesgos del entorno comprenden los elementos del país donde está ubicada la empresa, su naturaleza, la región y ciudad, además del sector, la industria y condiciones económicas, políticas, sociales y culturales (Mejía, 2006); los estratégicos son aquellos asociados a las amenazas y oportunidades transversales a la organización y relacionadas con las definiciones estratégicas, su filosofía de operación e imagen, tales como, visión, misión, valores, objetivos, diferenciación estratégica, entre otras (Castellanos, 2007); los operacionales están asociados a las amenazas y oportunidades transversales a la organización y su filosofía de operación (Castellanos, 2007) y los riesgos financieros hacen referencia a la incertidumbre asociada al rendimiento de la inversión debido a la posibilidad de que la empresa no pueda hacer frente a sus obligaciones financieras (Mascareñas, 2008). 


\section{METODOLOGÍA}

\section{Estudio de caso}

Se decide usar un estudio de caso al ser la forma más característica de las investigaciones cualitativas (Lozoya, 2006) que permite extraer conclusiones de fenómenos reales (Quintana, 2006); aunado a que no se cuenta con información que permita contrastación dada las escasas metodologías cualitativas para la evaluación de riesgos, entendiendo que el objetivo del estudio es diagnosticar la viabilidad del uso de la metodología ERM en microempresas.

Para este estudio se siguió la metodología establecida por Pérez, Campos y García (2013), que se fundamenta en el proceso establecido por el ERM para la evaluación de riesgos, que consiste en cuatro pasos: a) Identificación del contexto; b) identificación del riesgo; c) evaluación cualitativa; d) priorización del riesgo. Los dos primeros se encuentran en el apartado de metodología y los dos últimos, en la sección de resultados.

\section{a) Identificación del contexto}

La microempresa se define como la pequeña unidad socioeconómica, cuya creación no requiere de mucho capital (González, 2005), constituida entre 0 y 10 empleados, con un rango de monto de ventas anuales de hasta cuatro millones de pesos (DOF, 2009) para el caso mexicano.

Y como el caso en estudio pertenece al sector manufacturero, Carpenter (2009) establece que la característica principal de este sector es la transformación de las materias primas en un producto totalmente terminado que ya está en condiciones de ser destinado a la venta. Considerando la clasificación de INEGI (2011), esta microempresa pertenece al subsector 332 Fabricación de productos metálicos que inició operaciones en 1989 con la fabricación de piezas y productos bajo diseños, a partir de materia prima como el acero, perfiles, PTR y otros componentes; sus principales productos son autopartes para camiones y piezas de autobuses; sus principales actividades son el corte, doblez, soldadura, troquelado y barrenado y a nivel tecnológico busca actualizar sus procesos con equipo de medición y maquinaria para realizarlos más rápida y precisa; la empresa reporta crecimiento en ventas desde que inició operaciones.

\section{b) Identificación del riesgo}

La identificación del riesgo se llevó a cabo a través de una entrevista al dueño de la microempresa en estudio, en la cual se identificó la frecuencia (viabilidad) e impacto de los riesgos financieros, operativos, estratégicos y del entorno, riesgos considerados en el instrumento IEREM de Pérez, Campos y García (2013) para la evaluación de riesgos empresariales.

\section{RESULTADOS}

\section{c) Evaluación cualitativa}

Para la evaluación de los riesgos, Pérez, Campos y García (2013) proponen la evaluación cualitativa a partir de la viabilidad e impacto, que se obtiene a partir de la valoración que el empresario hizo a cada uno de los indicadores de riesgos, valores que son ubicados en el cuadro de evaluación de riesgos, en el cual se identifican cuatro cuadrantes, considerando el eje de las Y como viabilidad y el eje de las X de impacto (figura $\mathrm{N}^{\circ} 1$ ). 


\section{Figura $\mathrm{N}^{\circ} 1$}

\section{Cuadro de evaluación de riesgos}

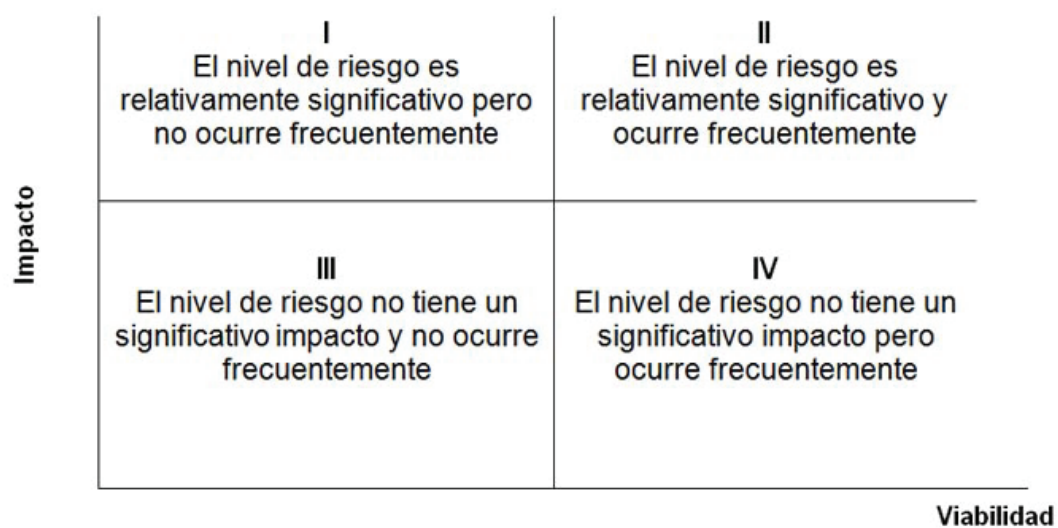

Fuente: ERM, adaptado por Pérez, Campos y García (2013)

Los datos recolectados en la empresa son los siguientes:

\section{Riesgos financieros}

\begin{tabular}{|l|c|c|}
\hline Riesgos financieros & Viabilidad & Impacto \\
\hline Estructura de capital & 1 & 1 \\
\hline Mercado & 1 & 1 \\
\hline Crédito & 2 & 2 \\
\hline Liquidez & 1 & 1 \\
\hline Tesorería & 1 & 1 \\
\hline Contabilidad & 1 & 1 \\
\hline
\end{tabular}

Fuente: Propia.

Ubicación de riesgos financieros en los cuadrantes:

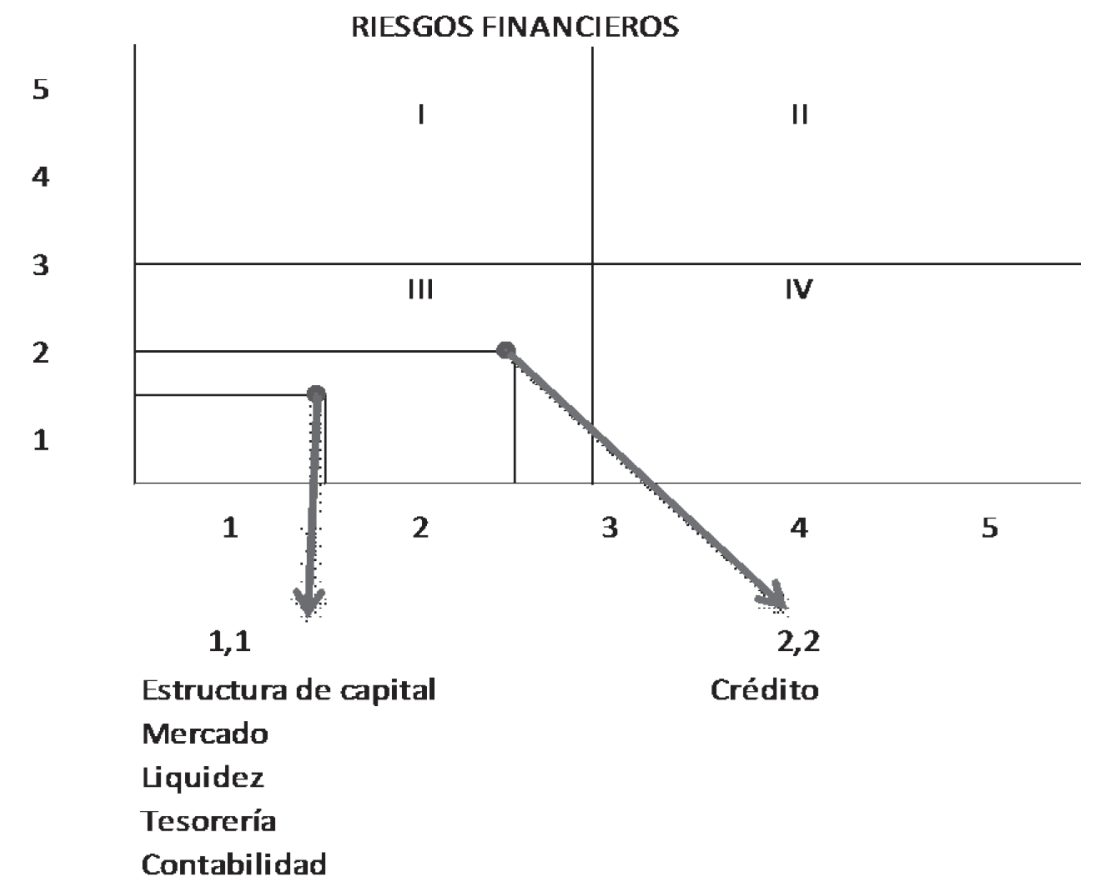

Fuente: Propia. 
Con base en la ubicación de estos riesgos en los cuadrantes, se observa que la empresa en estudio, en cuanto a riesgos financieros, se observa que todos los indicadores (liquidez, tesorería, estructura de capi- tal, mercado, contabilidad y crédito) se encuentran localizados en el cuadrante III, que implica que el nivel de riesgo no tiene un significativo impacto y no ocurre frecuentemente.

\section{Riesgos operativos}

\begin{tabular}{|l|c|c|}
\hline Riesgos operativos & Viabilidad & Impacto \\
\hline Fraude interno & 1 & 1 \\
\hline Fraude externo & 1 & 1 \\
\hline Personal & 1 & 1 \\
\hline Compras & 1 & 1 \\
\hline Proveedores & 2 & 2 \\
\hline Recepción y almacenaje & 1 & 1 \\
\hline Ventas & 1 & 1 \\
\hline Mercadotecnia & 1 & 1 \\
\hline Tecnología y sistemas de información & 1 & 1 \\
\hline Cumplimiento & 1 & 1 \\
\hline Ejecución y administración de procesos & 1 & 1 \\
\hline
\end{tabular}

Ubicación de riesgos operativos en los cuadrantes:

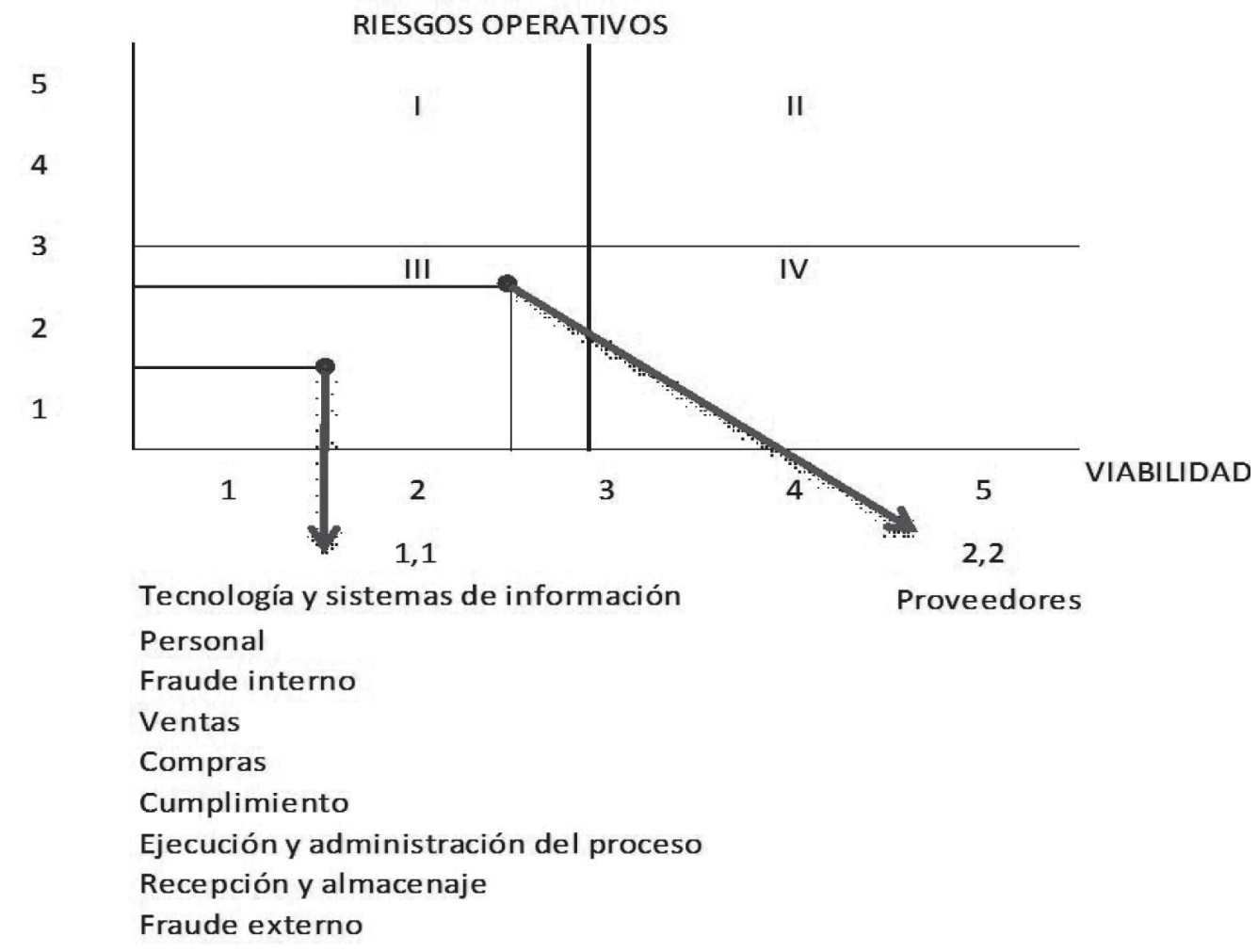

Fuente: Propia. 
Respecto a los riesgos operativos, se observa que en el cuadrante III se ubican todos los indicadores (fraude interno, fraude externo, personal, compras, recepción y almacenaje, ventas, mercadotecnia, tec- nología y sistemas de información, cumplimiento, ejecución y administración de los procesos), lo que representa que en la operación no tienen un significativo impacto y no ocurren frecuentemente.

\section{Riesgos estratégicos}

\begin{tabular}{|l|c|c|}
\hline Riesgos estratégicos & Viabilidad & Impacto \\
\hline Cambios del sector & 1 & 1 \\
\hline Cambio de tecnología & 2 & 1 \\
\hline Investigación y desarrollo & 1 & 1 \\
\hline Competencia & 2 & 2 \\
\hline Cambio de clientes & 1 & 1 \\
\hline Fusiones, adquisiciones o despojo & 1 & 1 \\
\hline Planificación & 1 & 1 \\
\hline Asignación de recursos & 1 & 1 \\
\hline Cambios en los precios & 1 & 1 \\
\hline Jerarquía y autoridad & 1 & 1 \\
\hline
\end{tabular}

Ubicación de riesgos estratégicos en los cuadrantes:

5

3

2

1

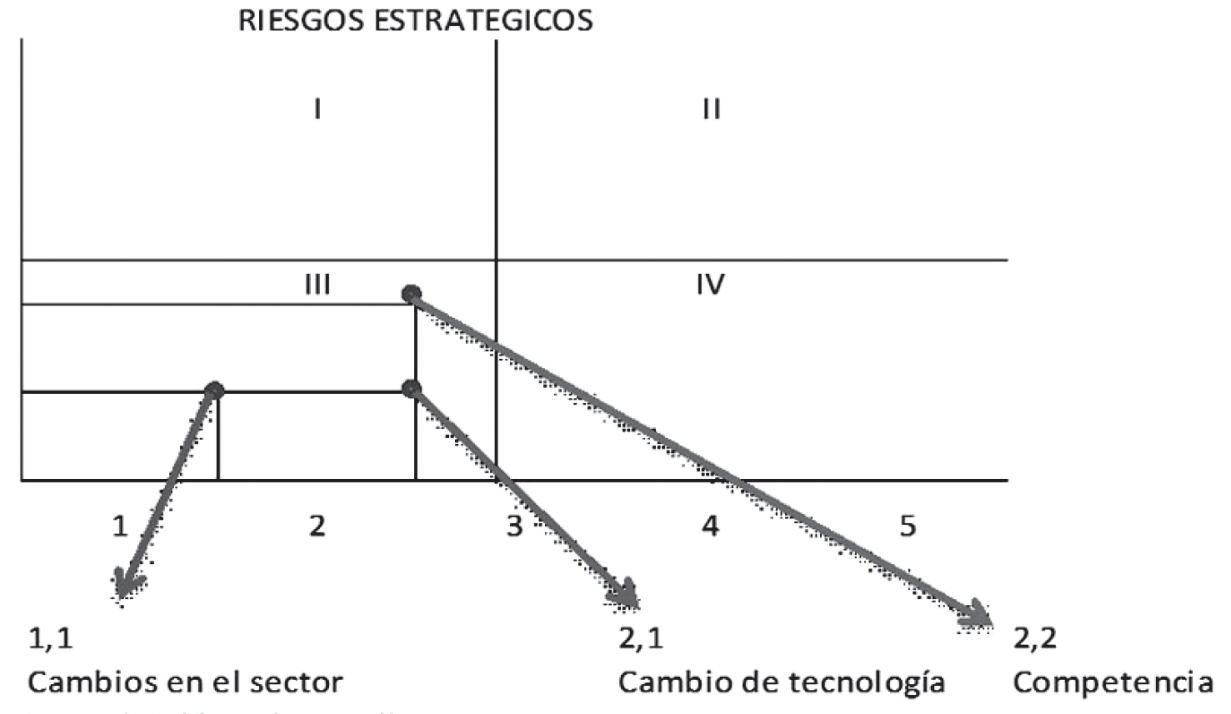

Investigación y desarrollo

Cambio en clientes

Fusiones, adquisiciones o despojo

Planificación

Asignación de recursos

Cambio de precios

Jerarquía y autoridad

Fuente: Propia. 
En el cuadrante de riesgos estratégicos se observa que todos los indicadores se localizan dentro del cuadrante III, por lo que estos riesgos no tienen un significativo impacto y no ocurren frecuentemente.

\section{Riesgos del entorno}

\begin{tabular}{|l|c|c|}
\hline Riesgos del entorno & Viabilidad & Impacto \\
\hline Patrimonio & 1 & 1 \\
\hline Productos y servicios & 1 & 1 \\
\hline Inseguridad & 1 & 1 \\
\hline Conflictos laborales & 1 & 1 \\
\hline Responsabilidad & 1 & 1 \\
\hline Desastres naturales & 1 & 1 \\
\hline Reputación & 2 & 2 \\
\hline
\end{tabular}

Ubicación de riesgos del entorno en los cuadrantes:

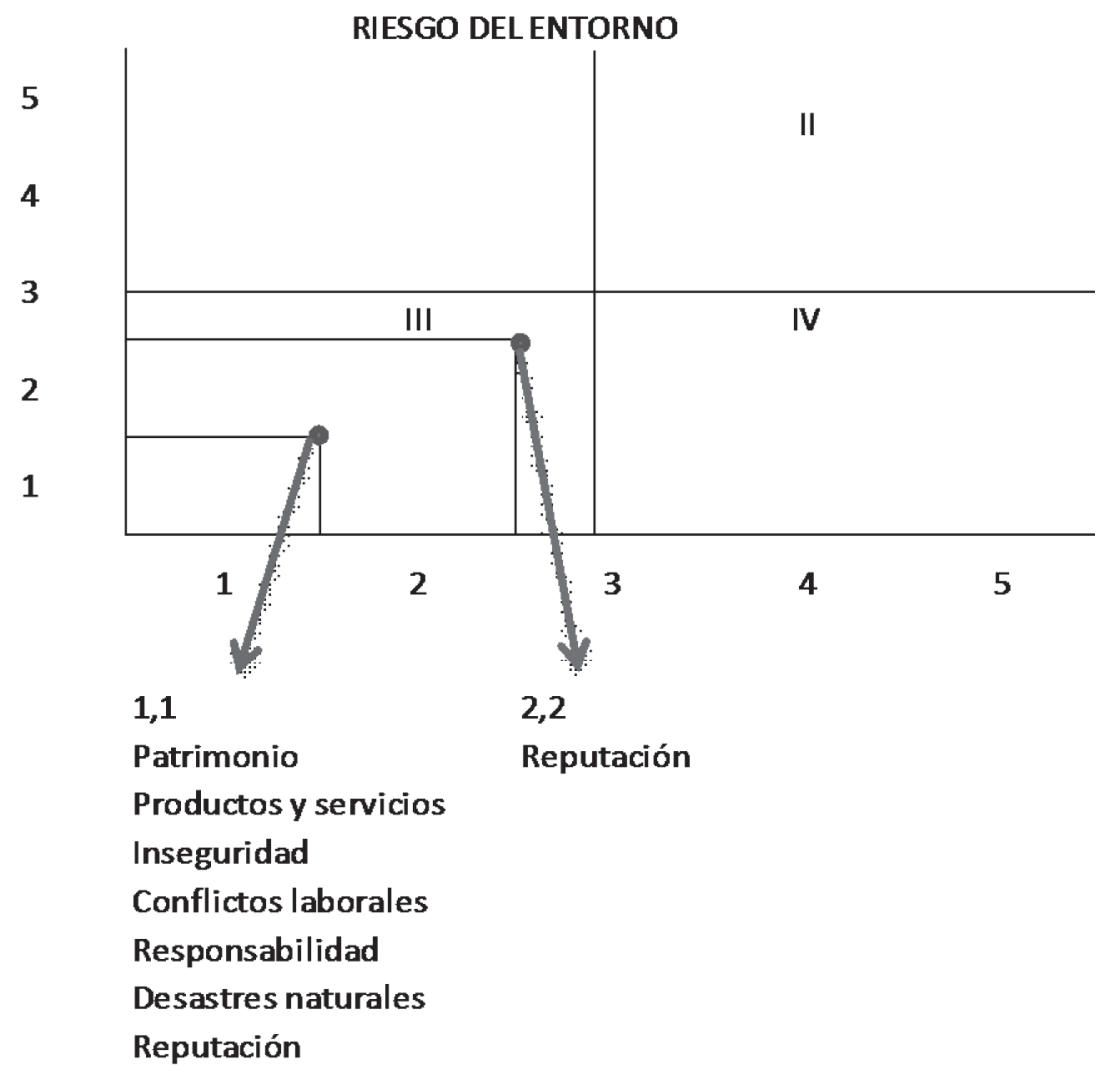

Fuente: Propia.

Finalmente, en cuanto a los riesgos del entorno, se observa que la empresa también se ubica en el cuadrante III, donde el nivel de riesgo no tiene un significativo impacto y no ocurre frecuentemente.

\section{d) Priorización del riesgo}

El siguiente paso de la evaluación es realizar el ranking de riesgos, para lo cual se transforman los valores de viabilidad e impacto en una escala de 10 para valorarlos a través de la multiplicación de la viabilidad (F) por el impacto (I), obteniendo los siguientes resultados: 


\begin{tabular}{|c|c|c|c|c|}
\hline Riesgo identificado & $\begin{array}{l}\text { Viabilidad } \\
\quad(F)\end{array}$ & $\begin{array}{c}\text { Impacto } \\
\text { (I) }\end{array}$ & $\begin{array}{c}\text { Valoración } \\
\text { de riesgo } \\
\left(\mathbf{F}^{*} \mathbf{I}\right)\end{array}$ & Clasificación \\
\hline \multicolumn{5}{|c|}{ Financieros } \\
\hline Estructura de capital & 2 & 2 & 4 & 3 \\
\hline Mercado & 2 & 2 & 4 & 3 \\
\hline Crédito & 4 & 4 & 16 & 1 \\
\hline Liquidez & 2 & 2 & 4 & 3 \\
\hline Tesorería & 2 & 2 & 4 & 3 \\
\hline Contabilidad & 2 & 2 & 4 & 3 \\
\hline \multicolumn{5}{|c|}{ Operativos } \\
\hline Fraude interno & 2 & 2 & 4 & 3 \\
\hline Fraude externo & 2 & 2 & 4 & 3 \\
\hline Personal & 2 & 2 & 4 & 3 \\
\hline Compras & 2 & 2 & 4 & 3 \\
\hline Proveedores & 4 & 4 & 16 & 1 \\
\hline Recepción y almacenaje & 2 & 2 & 4 & 3 \\
\hline Ventas & 2 & 2 & 4 & 3 \\
\hline Mercadotecnia & 2 & 2 & 4 & 3 \\
\hline Tecnología y sistemas de información & 2 & 2 & 4 & 3 \\
\hline Cumplimiento & 2 & 2 & 4 & 3 \\
\hline Ejecución y administración de procesos & 2 & 2 & 4 & 3 \\
\hline \multicolumn{5}{|c|}{ Estratégicos } \\
\hline Cambios del sector & 2 & 2 & 4 & 3 \\
\hline Cambio de tecnología & 4 & 2 & 8 & 2 \\
\hline Investigación y desarrollo & 2 & 2 & 4 & 3 \\
\hline Competencia & 4 & 4 & 16 & 1 \\
\hline Cambio de clientes & 2 & 2 & 4 & 3 \\
\hline Fusiones, adquisiciones o despojo & 2 & 2 & 4 & 3 \\
\hline Planificación & 2 & 2 & 4 & 3 \\
\hline Asignación de recursos & 2 & 2 & 4 & 3 \\
\hline Cambios en los precios & 2 & 2 & 4 & 3 \\
\hline Jerarquía y autoridad & 2 & 2 & 4 & 3 \\
\hline \multicolumn{5}{|c|}{ Del entorno } \\
\hline Patrimonio & 2 & 2 & 4 & 3 \\
\hline Productos y servicios & 2 & 2 & 4 & 3 \\
\hline Inseguridad & 2 & 2 & 4 & 3 \\
\hline Conflictos laborales & 2 & 2 & 4 & 3 \\
\hline Responsabilidad & 2 & 2 & 4 & 3 \\
\hline Desastres naturales & 2 & 2 & 4 & 3 \\
\hline Reputación & 4 & 4 & 16 & 1 \\
\hline
\end{tabular}

Fuente: Propia. 
Como se observa en el ranking de riesgos, los 4 riesgos que tienen mayor nivel de exposición son: crédito, proveedores, competencia, reputación, con un valor de 16 , indicadores que pertenecen a cada uno de los riesgos estudiados; el de cambio de tecnología se encuentra en segundo lugar con un valor de 8 , dentro de los riesgos estratégicos y finalmente, con una valoración de 4 están todos los demás.

De tal manera, que aunque los riesgos en la empresa en estudio no tienen un impacto significativo ni ocurren frecuentemente, es necesario que se tenga atención en los indicadores con valoración de 16 , pues indican que son los que más alto nivel de exposición tienen.

\section{CONCLUSIONES}

1. La metodología ERM para la evaluación de riesgos en microempresa es útil, aplicable y a partir de información cualitativa, ubica a los riesgos en niveles que permiten visualizar a la empresa en un contexto integral.

2. De acuerdo al procedimiento de evaluación de riesgos aplicado, los riesgos empresariales en la microempresa en estudio se ubican en el rango más bajo de viabilidad e impacto, ubicados en el cuadrante III con impacto poco significativos y poco frecuentes.

3. En cuanto al ranking de riesgos, se observan con las valoraciones más altas de exposición crédito (riesgo financiero), proveedores (riesgos operativos), competencia (estratégico) y reputación (del entorno).

4. Considerando el enfoque del ERM, los niveles de exposición de riesgos mostrados en la microempresa hacen inferir que el dueño los ha gestionado adecuadamente minimizándolos en una forma empírica.

\section{REFERENCIAS BIBLIOGRÁFICAS}

1. Arnaiz, C (2013). Modelo ERM. 04 de septiembre de 2013, en http://www.aec.es/web/guest/ centro-conocimiento/modelo-erm

2. Caicedo, W. (2010) Gestión de riesgos corporativos. 09 de septiembre de 2013, en http://
es.scribd.com/doc/33482297/Gestion-de-Riesgos-Corporativos-ERM

3. Carpenter, M. (2009) Sector manufacturero. 11 de septiembre de 2013, en http://www.ehowenespanol.com/son-sectores-primario-secundario-terciario-info_147360/

4. Castellanos, J. (Junio - julio 2007) Gestión del riesgo empresarial. Qualinet Surlatina Gestion, pp. 36-38.

5. DOF (2013).Reglas de operación del fondo de apoyo para la micro, pequeña y mediana empresa (PyME) para el ejercicio fiscal 2013. Diario Oficial de la Federación.

6. González, T. (2005). Problemas en la definición de microempresa. Revista Venezolana de Gerencia.10, 31, pp.408 - 422.

7. Guzmán, O. (2013) ¿Qué es el riesgo empresarial? Consultado el 2 de septiembre de 2013, en http://pyme.lavoztx.com/qu-es-el-riesgo-empresarial-4339.html

8. INEGI (2011). Clasificación del sector manufacturero. Recuperado el 16 de septiembre de 2013, de www.inegi.gob.mx

9. Koprinarov, B. (2005). El riesgo empresarial y su gestión. Consultado el 15 de noviembre de 2013, de http://www.analitica.com/va/economia/ opinion/5753437.asp

10.Lozoya, M. (Agosto de 2006). Paradigmas de investigación cualitativa. Instituto Tecnologico de Sonora. Recuperado el 30 de septiembre de 2013, de http://biblioteca.itson.mx/oa/educacion/oa2/ParadigmasInvestigacionCualitativa/ index.htm

11.Mascareñas, J. (2008) Riesgo económico y financiero. Monografía sobre finanzas corporativas. Universidad Complutense de Madrid: España.

12.Mejía, R. (2006). Definición y tipos de riesgos. Consultorio Contable, Universidad EAFIT: Colombia.

13.ISACA (2009). The risk IT framework: Autor.

14.Pérez, S., Campos, C. y García. F. (2013). Evaluación de riesgos empresariales en microempresas a través del IEREM. Segundo Encuentro Nacional 
de Cuerpos Académicos "Rumbo a la conformación de redes de colaboración en competitividad". UAEH, UAEM, UAT e ITESA. Hidalgo, México.
15.Quintana, T.L. (2006). Métodos y técnicas de investigación, México: Mc Graw Hill

16.Soldano, A. (2009) Síntesis temática de ERM. CONAE, OEA y DSS: Argentina. 